\title{
Avaliação de características de desempenho e de carcaça de codornas de corte por meio da análise de componentes principais
}

[Evaluation of production and carcass traits of meat - type quails by principal components analysis]

\author{
C.D.S. Leite ${ }^{1}$, G.S.S. Corrêa ${ }^{2}$, L. Barbosa ${ }^{3}$, A.L.P. Melo ${ }^{4}$, M. Yamaki ${ }^{4}$, M.A. Silva ${ }^{2}$, R.A. Torres ${ }^{4}$ \\ ${ }^{1}$ Departamento de Zootecnia - UNESP - Jaboticabal, SP \\ ${ }^{2}$ Escola de Veterinária - UFMG - Belo Horizonte, MG \\ ${ }^{3}$ Núcleo de Zootecnia - UFSE - Aracajú, SE \\ ${ }^{4}$ Departamento de Zootecnia - UFV - Viçosa, MG
}

\begin{abstract}
RESUMO
Foram utilizados dados de 288 codornas de corte (Coturnix coturnix coturnix) para avaliar a possibilidade de resumir a informação contida no complexo de variáveis originais, eliminando-se variáveis inexpressivas por meio da técnica de componentes principais. Foram registrados o peso vivo (PVIVO) e pesos do peito (PPEITO), das coxas (PCOXA), da gordura abdominal (GA), das vísceras comestíveis (fígado, moela e coração) (FIG, MOELA e CORA) e da carcaça eviscerada (PCEVIS). As carcaças foram secas e trituradas para a avaliação do teor matéria seca (MS), gordura (GORD) e proteína bruta (PB). Dos 11 componentes principais, sete $(63,6 \%)$ apresentaram variância menor que 0,7 (autovalor inferior a 0,7 ), sendo sugeridas para descarte, respectivamente, em ordem de menor importância, para explicar a variação total das seguintes variáveis: PCEVIS, PPEITO, PCOXA, CORA, FIG MOELA e GORD. Com base nos resultados, recomenda-se manter as seguintes variáveis em experimentos futuros: PVIVO, MS, PB e GA.
\end{abstract}

Palavras-chave: codorna, carcaça, descarte de variável

\begin{abstract}
Records on 288 meat type quails (Coturnix coturnix coturnix) were used to identify independent and informative variables by eliminating inexpressive variables by means of principal component analysis. The following performance traits were recorded: live body weight (LBW), eviscerated carcass weight $(C W)$, whole leg weight $(W L W)$, breast weight $(B W)$, abdominal fat pad weight $(F W)$, and giblets weight (liver, gizzard, and heart) ( $L I W, G W$, and $H W$ ). The carcasses were also dried and grounded to evaluate dry matter (DM), fat $(F A, T)$ and crude protein $(C P)$ contents. Out of 11 principal components, seven (63.6\%) showed variance lower than 0.7 (eigenvalue lower than 0.7). The following traits were discarded because they showed inexpressive contribuitions for the total variation: $C W, B W, W L W, H W, L I W, G W$, and FAT. Based on the results, it is recommended to record the following variables in future experiments: $L B W, D M, C P$, and $F W$.
\end{abstract}

Keywords: quail, carcass, discard of variable

\section{INTRODUÇÃO}

A criação de codornas para produção de carne é uma boa alternativa para obtenção de proteína de origem animal, por ser um animal de pequeno e rápido crescimento. Entretanto, pouco se conhece sobre o potencial produtivo de codornas de corte no Brasil e ainda há poucas linhagens nacionais com características produtivas adequadas à produção de carne. As codornas de corte apresentam maior porte e são mais indicadas para a produção de carne por obterem maiores pesos na mesma idade de abate do que as codornas japonesas, que têm sido utilizadas também para a produção de carne (Corrêa et al., 2006).

Recebido em 2 de julho de 2008 
O material genético disponível para produção de codornas para carne no Brasil é pouco adequado em razão da utilização de codornas japonesas ou "italianas", selecionadas para a produção de ovos. Essas aves apresentam características pouco adequadas para a produção de carne. Assim, há necessidade de se obter material genético especializado, com maior peso e melhor conformação de carcaça ideal para o atendimento dos mercados interno e de exportação (Garcia, 2002). Para se obter material genético de qualidade, são necessários programas de melhoramento bem fundamentados, embasados em parâmetros genéticos acurados e precisos (Dionello et al., 2008). No entanto, em muitas situações, os pesquisadores geram um considerável acréscimo de trabalho ao avaliarem um maior número de características. Embora não haja limite, na prática, para o número de características que podem ser avaliadas nos experimentos, é importante examinar se algumas poderiam ser desconsideradas na coleta das observações ou nas análises estatísticas.

Quando se dispõe de grande número de descritores, é possível que muitos deles sejam redundantes, tornando-se útil a sua eliminação, porque, além de pouco informativos, ocorre acréscimo no trabalho de avaliação (Jolliffe, 1972, 1973). Assim, o descarte das variáveis pode ser feito por meio de componentes principais, que têm como principal objetivo resumir a informação contida no complexo de variáveis originais, eliminando as informações redundantes existentes em decorrência da correlação entre estas (Khattree e Naik, 2000).

Este trabalho teve como objetivo reduzir a dimensionalidade do conjunto original de variáveis, eliminando as informações redundantes, e viabilizando a recomendação de variáveis a serem avaliadas em experimentos futuros.

\section{MATERIAL E MÉTODOS}

O experimento foi realizado com 288 codornas de corte (Coturnix coturnix coturnix), de ambos os sexos, alojadas em baterias metálicas. As dietas foram formuladas baseando-se nas informações de composições dos ingredientes apresentadas por Rostagno et al. (2000). Para atender às exigências nutricionais das codornas, usou-se a recomendação do NRC (Nutrient..., 1994), exceto para proteína bruta (PB) e energia metabolizável (EM). As dietas foram fornecidas à vontade.

Foram estudados peso vivo (PVIVO) no período inicial (7-21 dias de idade), no período complementar (22-42 dias de idade) e no período total (7-42 dias de idade). No $42^{\underline{o}}$ dia de idade, após jejum de sólidos de oito horas, um macho e uma fêmea por unidade experimental foram pesados, marcados por meio de lacre e anel em um dos pés e abatidos em abatedouro. A seguir, foram depenados, eviscerados e submetidos aos cortes para avaliações do rendimento da carcaça, em relação ao PVIVO, dos pesos do peito (PPEITO), coxas (PCOXA), gordura abdominal (GA), vísceras comestíveis, fígado, moela $\mathrm{e}$ coração, (FIG, MOELA e CORA) e da carcaça eviscerada (PCEVIS). Após as avaliações de peso e rendimento, as carcaças foram secas e trituradas para a avaliação do teor matéria seca (MS), gordura (GORD) e proteína bruta (PB).

Tendo em vista o grande número de variáveis medidas em unidades diferentes, foi necessária a padronização dessas variáveis $\mathrm{Xj}(\mathrm{j}=1,2, \ldots, \mathrm{p})$; nesse caso, a estrutura de dependência de $\mathrm{Xj}$ foi dada pela matriz de correlação R. Os dados foram submetidos à análise de componentes principais, os quais foram obtidos com base nas seguintes expressões:

$|R-\lambda I|=0 \rightarrow$ fornece os autovalores $\lambda_{1}, \lambda_{2}, \ldots, \lambda \mathrm{p}$;

$[R-\lambda I] a_{i}=\phi \rightarrow$ fornece os autovetores $\mathrm{a}_{1}, \mathrm{a}_{2}$, $\ldots \mathrm{a}_{\mathrm{p}}$; em que:

$R=$ matriz de correlação entre as características avaliadas;

$\lambda_{i}=$ autovalores da matriz $\mathrm{R}$;

$a_{i}=$ autovetor associado ao autovalor $\lambda_{i}$;

$I=$ matriz identidade de ordem $\mathrm{p}(\mathrm{p}=$ número de características);

$\phi=$ é um vetor nulo, de dimensão p x 1

Os autovetores $a_{i}$ foram normalizados de modo

a obter $\underset{\sim}{\mathrm{a}_{\mathrm{i}}^{*}}$ tal que $\underset{\sim}{\mathrm{a}_{\mathrm{i}}^{\prime *}}{\underset{\sim}{\mathrm{i}}}^{*}=1$ para $i=1,2, \ldots, \mathrm{p} \mathrm{e}$

$\mathrm{a}_{i}^{\prime *} \mathrm{a}_{\mathrm{j}}=0$ para $i \neq j$.

Segundo Morrison (1976), os componentes principais são combinações lineares das variáveis 
originais e são estimados de forma que os primeiros componentes principais associados aos maiores autovalores retenham o maior percentual da variância total. Cada variável original está associada ao componente principal por meio de um coeficiente de ponderação, assim os coeficientes de maior valor absoluto nos componentes principais de menor autovalor são passíveis de descarte. Para o descarte de variáveis, a variável que possuir maior correlação no componente principal de menor autovalor (menor variância) deve ser menos importante para explicar a variância total e, portanto, passível de descarte (Regazzi, 2002). Com base no princípio de que a importância ou a variância dos componentes principais decresce do primeiro para o último, tem-se que os últimos componentes explicam uma fração muito pequena da variância total. Então, a variável de maior coeficiente no componente de menor autovalor deve ser a menos importante para explicar a variância total e, portanto, passível de descarte (Pereira, 1989).

A técnica dos componentes principais tem a vantagem adicional de avaliar a importância de cada característica estudada sobre a variação total disponível entre os acessos avaliados, possibilitando o descarte das características menos discriminantes, por já estarem correlacionadas com outras variáveis ou pela sua invariância (Daher et al., 1997). A razão para isso é que variáveis altamente correlacionadas com os componentes principais de menor variância representam variação praticamente insignificante.

O critério do número de variáveis descartadas foi, conforme recomendações de Jolliffe (1973), baseado em dados simulados e reais, com a análise de componentes principais a partir da matriz de correlação. Este critério estabelece que o número de variáveis descartadas deve ser igual ao número de componentes cuja variância (autovalor) é inferior a 0,7. Todas as análises foram feitas utilizando o programa SAS/1999.

\section{RESULTADOS E DISCUSSÃO}

Os resultados obtidos para os componentes principais, seus respectivos autovalores e porcentagens da variância total explicada por cada um são apresentados na Tab.1.

Tabela1. Componentes principais (CP), autovalores $\left(\lambda_{i}\right)$ e porcentagem da variância explicada pelos componentes $(\% \mathrm{VCP})$ nas características de carcaça de codornas

\begin{tabular}{cccc}
\hline Componentes principais & $\lambda_{i}$ & \% VCP & \% VCP (acumulada) \\
\hline $\mathrm{CP}_{1}$ & 4,7143 & 42,86 & 42,86 \\
$\mathrm{CP}_{2}$ & 1,6374 & 14,89 & 57,74 \\
$\mathrm{CP}_{3}$ & 1,0486 & 9,53 & 67,28 \\
$\mathrm{CP}_{4}$ & 0,8500 & 7,73 & 75,00 \\
$\mathrm{CP}_{5}$ & 0,6546 & 5,95 & 80,95 \\
$\mathrm{CP}_{6}$ & 0,5871 & 5,34 & 86,29 \\
$\mathrm{CP}_{7}$ & 0,5366 & 4,88 & 91,17 \\
$\mathrm{CP}_{8}$ & 0,4557 & 4,14 & 95,31 \\
$\mathrm{CP}_{9}$ & 0,3089 & 2,81 & 98,12 \\
$\mathrm{CP}_{10}$ & 0,1388 & 1,26 & 99,38 \\
$\mathrm{CP}_{11}$ & 0,0680 & 0,62 & 100 \\
\hline
\end{tabular}

Os quatro primeiros componentes principais explicaram $75 \%$ da variação total. Dos 11 componentes principais, sete $(63,6 \%)$ apresentaram variância menor que 0,7 (autovalor inferior a 0,7). Dessa forma, essas características podem ser descartadas de acordo com o critério de Jolliffe $(1972,1973)$.

Abreu et al. (1999), ao trabalharem com produção de ovos de matrizes de frango de corte, utilizando componentes principais, observaram que os dois primeiros componentes principais explicaram mais de $98 \%$ da variação total disponível entre as médias de cruzamentos disponíveis. Barbosa et al. (2005a), ao avaliarem 11 características de desempenho em suínos, concluíram que os três primeiros componentes principais contribuíram com $67,9 \%$ da variação total. 
Pinto et al. (2006), ao utilizarem a técnica de componentes principais para obter uma combinação linear das características de performance e carcaça de frangos, a fím de reduzir a complexidade dos índices de seleção, concluíram que os cinco primeiros componentes principais explicaram, juntos, uma alta porcentagem (93\%) da variância total.

As sete variáveis que apresentaram maiores coeficientes, em valor absoluto, a partir do último componente principal, foram descartadas, conforme apresentado na Tab. 2. O componente principal 5 (PCOXA) não eliminou nenhuma variável, uma vez que a característica FIG havia sido eliminada anteriormente.
Yamaki et al. (2005), ao avaliarem a possibilidade de descarte de variáveis de produção, por meio de componentes principais, de três linhas de matrizes de frango de corte, encontraram resultados semelhantes, ao concluírem que, das 12 variáveis em estudo, sete foram redundantes, podendo ser descartadas.

Em um estudo de características de carcaça de suínos, Barbosa et al. (2005b) relataram a possibilidade de descarte de $51,5 \%$ das 33 variáveis analisadas.

De acordo com Barbosa (2005a), as variáveis que apresentaram maiores correlações com o último componente principal devem ser descartadas por apresentarem contribuição insignificante da variação total.

Tabela 2. Coeficientes de ponderação das características de carcaça de codornas com os componentes principais descartados em ordem de menor importância

\begin{tabular}{ccccccccc}
\multicolumn{2}{c}{ Característica } & \multicolumn{7}{c}{ Coeficiente } \\
\cline { 2 - 9 } & $\mathrm{CP}_{4}$ & $\mathrm{CP}_{5}{ }^{1}$ & $\mathrm{CP}_{6}$ & $\mathrm{CP}_{7}$ & $\mathrm{CP}_{8}$ & $\mathrm{CP}_{9}$ & $\mathrm{CP}_{10}$ & $\mathrm{CP}_{11}$ \\
\hline PVIVO & 0,0680 & $-0,1152$ & $-0,0213$ & $-0,1583$ & 0,0283 & 0,0015 & 0,5934 & $-0,6383$ \\
PCEVIS & 0,1095 & $-0,3226$ & $-0,1489$ & $-0,2118$ & 0,1194 & 0,1239 & 0,3147 & $\mathbf{0 , 7 1 3 4}$ \\
GA & 0,0444 & 0,2746 & $-0,1375$ & 0,4356 & $-0,4652$ & 0,4843 & $-0,0729$ & 0,0140 \\
FIG & $-0,1660$ & 0,4773 & 0,1573 & $\mathbf{- 0 , 6 0 7 8}$ & $-0,3854$ & $-0,0458$ & $-0,1472$ & 0,0776 \\
PCOXA & $-0,3139$ & $-0,2284$ & 0,2787 & 0,3091 & $-0,2375$ & $\mathbf{- 0 , 6 6 9 1}$ & $-0,0982$ & 0,0696 \\
PPEITO & 0,1787 & $-0,3300$ & 0,0190 & $-0,1845$ & 0,2751 & 0,1673 & $\mathbf{- 0 , 7 0 4 2}$ & $-0,2352$ \\
MOELA & 0,2675 & 0,0370 & $\mathbf{0 , 5 0 2 4}$ & 0,4075 & 0,0923 & 0,1752 & 0,0614 & 0,0574 \\
MS & $-0,4512$ & 0,0340 & 0,4677 & $-0,1240$ & 0,2792 & 0,3035 & 0,0908 & $-0,0083$ \\
PB & 0,0822 & 0,3625 & 0,3906 & 0,0153 & 0,3639 & $-0,0272$ & 0,0464 & 0,1154 \\
GORD & $\mathbf{0 , 6 6 7 5}$ & 0,3106 & 0,0449 & $-0,0758$ & 0,0726 & $-0,3550$ & 0,0087 & 0,0227 \\
CORA & $-0,3098$ & 0,4336 & $-0,4795$ & 0,2322 & $\mathbf{0 , 5 1 3 6}$ & $-0,1506$ & $-0,0450$ & 0,0075 \\
\hline PVIVO:
\end{tabular}

PVIVO: peso vivo; PCEVIS: peso de carcaça eviscerada; GA: gordura abdominal; FIG: peso de fígado; PCOXA: peso de coxa; PPEITO: peso do peito; MOELA: peso de moela; MS: matéria seca da carcaça; PB: teor de proteína bruta da carcaça; GORD: teor de gordura da carcaça; CORA: peso de coração.

${ }^{1}$ No quinto componente principal, não houve sugestão para descarte, uma vez que o maior coeficiente foi de uma característica passível de descarte em um componente anterior.

As variáveis sugeridas para descarte foram, respectivamente, em ordem de menor importância para explicar a variação total: PCEVIS, PPEITO, PCOXA, CORA, FIG, MOELA e GORD (Tab. 2). Com base nestes resultados, recomendam-se as seguintes variáveis para serem mantidas em experimentos futuros: PVIVO, MS, PB e GA.

As características sugeridas para descarte apresentaram correlação linear simples significativas com as demais, ou seja, são redundantes. Por outro lado, as variáveis selecionadas apresentaram menor correlação entre si, conforme apresentado na Tab. 3. Deste modo, cada característica selecionada deve ser responsável por um tipo de informação biológica exclusiva, e a ação conjunta delas será complementar para a descrição geral do indivíduo ou população estudadas. 
Leite et al.

Tabela 3. Coeficientes de correlação simples entre as características de carcaça de codornas

\begin{tabular}{ccccccccccccc}
\hline & PVIVO & PCEVIS & GA & FIG & PCOXA & PPEITO & MOELA & MS & PB & GORD & CORA \\
\hline PVIVO & 1,0000 & 0,9009 & 0,6098 & 0,6098 & 0,6779 & 0,8333 & 0,4083 & $-0,1522$ & 0,1381 & 0,0142 & 0,5474 \\
PCEVIS & 0,9009 & 1,0000 & 0,5672 & 0,5032 & 0,6252 & 0,8791 & 0,3491 & $-0,0961$ & 0,0026 & 0,0623 & 0,5125 \\
GA & 0,6098 & 0,5672 & 1,0000 & 0,3559 & 0,5646 & 0,5235 & 0,1665 & 0,0266 & 0,0929 & 0,1918 & 0,4381 \\
FIG & 0,6098 & 0,5032 & 0,3559 & 1,0000 & 0,4099 & 0,4599 & 0,3935 & $-0,0957$ & 0,0634 & $-0,0733$ & 0,4703 \\
PCOXA & 0,6779 & 0,6252 & 0,5646 & 0,4099 & 1,0000 & 0,5982 & 0,2854 & 0,099 & 0,0431 & $-0,0461$ & 0,4386 \\
PPEITO & 0,8333 & 0,8791 & 0,5235 & 0,4599 & 0,5982 & 1,0000 & 0,3856 & $-0,0923$ & 0,0977 & 0,0739 & 0,4554 \\
MOELA & 0,4083 & 0,3491 & 0,1665 & 0,3935 & 0,2854 & 0,3856 & 1,0000 & $-0,1931$ & $-0,0671$ & $-0,0129$ & 0,3245 \\
MS & $-0,1522$ & $-0,0961$ & 0,0266 & $-0,0957$ & 0,099 & $-0,0923$ & $-0,1931$ & 1,0000 & $-0,332$ & 0,3012 & 0,0097 \\
PB & 0,1381 & 0,0026 & 0,0929 & 0,0634 & 0,0431 & 0,0977 & $-0,0671$ & $-0,332$ & 1,0000 & $-0,2572$ & $-0,0656$ \\
GORD & 0,0142 & 0,0623 & 0,1918 & $-0,0733$ & $-0,0461$ & 0,0739 & $-0,0129$ & 0,3012 & $-0,2572$ & 1,0000 & $-0,0135$ \\
CORA & 0,5474 & 0,5125 & 0,4381 & 0,4703 & 0,4386 & 0,4554 & 0,3245 & 0,0097 & $-0,0656$ & $-0,0135$ & 1,0000
\end{tabular}

PVIVO: peso vivo; PCEVIS: peso de carcaça eviscerada; GA: gordura abdominal; FIG: peso de fígado; PCOXA: peso de coxa; PPEITO: peso do peito; MOELA: peso de moela; MS: matéria seca da carcaça; PB: teor de proteína bruta da carcaça; GORD: teor de gordura da carcaça; CORA: peso de coração.

\section{CONCLUSÕES}

A utilização da análise de componentes principais no descarte de variáveis mostrou-se efetiva e o menor número de variáveis necessárias para explicar a variação total observada resultou em importante economia de tempo e recursos em experimentos futuros, sem perda significativa de informação.

\section{REFERÊNCIAS BIBLIOGRÁFICAS}

ABREU, V.M.N.; SILVA, M.A.; CRUZ, C.D. et al. Capacidade de combinação de características de produção de ovos de linhagens de matrizes de corte, usando a técnica de componentes principais. Rev. Bras. Zootec., v.28, p.250-257, 1999.

BARBOSA, L.; LOPES, P.S.; REGAZZI, A.J. et al. Avaliação de características de carcaça de suínos utilizando-se a análise dos componentes principais. Rev. Bras. Zootec., v.34, supl., p.2209-2217, 2005a.

BARBOSA, L.; LOPES, P.S.; REGAZZI, A.J. et al. Seleção de variáveis de desempenho de suínos por meio da análise de componentes principais. Arq. Bras. Med. Vet. Zootec., v.57, p.805-810, 2005b.
CORRÊA, G.S.S.; SILVA, M.A.; CORRÊA, A.B. et al. Exigência de metionina + cistina para codornas de corte em crescimento. Arq. Bras. Med. Vet. Zootec., v.58, p.414-420, 2006.

DAHER, R.F.; MORAES, C.F.; CRUZ, C.D. Seleção de caracteres morfológicos em capimelefante (Pennisetum purpureum Schum.). Rev. Bras. Zootec., v.26, p.247-259, 1997.

DIONELLO, N.J.L.; CORREA, G.S.S.; SILVA, M.A. et al. Estimativas da trajetória genética do crescimento de codornas de corte utilizando modelos de regressão aleatória. Arq. Bras. Med. Vet. Zootec., v.60, p.454-460, 2008

GARCIA, E.A. Codornas para produção de carne. In: SIMPÓSIO INTERNACIONAL DE COTURNICULTURA, 1., 2002, Lavras. Anais... Lavras: UFLA, 2002. 97p.

JOLLIFFE, I.T. Discarding variables in a principal component analysis. I. Artificial data. Appl. Stat., v.21, p.160-173, 1972.

JOLLIFFE, I.T. Discarding variables in a principal component analysis. II. Real data. Appl. Stat., v.22, p.21-31, 1973.

KHATTREE, R.; NAIK, D.N. Multivariate data reduction and discrimination with SAS software. Cary: SAS Institute, 2000. 574p. 
MORRISON, D.F. Multivariate statistical methods. 2.ed. Singapore: McGraw Hill, 1976. $415 \mathrm{p}$.

NUTRIENT requirements of poultry. 9.ed. Washington: National Academy of Sciences, 1994. p.44-45.

PEREIRA, A.V. Utilização de análise multivariada na caracterização de germoplasma de mandioca (Manihot esculenta Crantz). 1989. 180f. Dissertação (Mestrado) - Escola Superior de Agricultura "Luiz de Queiroz", Universidade de São Paulo, Piracicaba.

PINTO, L.F.B.; PACKER, I.U.; MELO, C.M.R. et al. Principal components analysis applied to performance and carcass traits in the chicken. Anim. Res., v.55, p.419-425, 2006.

REGAZZI, A. J. Análise multivariada: notas de aula. Viçosa: UFV, 2002. ROSTAGNO, H.S. Tabelas brasileiras para aves e suinos: composição de alimentos e exigências nutricionais. Viçosa: UFV, 2000. 141p.

YAMAKI, M.; BARBOSA, L.; PAIVA, A.L.C. et al. Avaliação de características de produção de aves de corte por meio de componentes principais. In: REUNIÃO ANUAL DA SOCIEDADE BRASILEIRA DE ZOOTECNIA. 42., 2005, Goiânia. Anais... Goiânia: SBZ, 2005. 